\title{
In silico epitope prediction, expression and functional analysis of Per a 10 allergen from the American cockroach
}

\author{
XUNLIANG TONG ${ }^{1 *}$, MIAO GUO $^{2 *}$, MIN JIN $^{2 *}, \mathrm{HAO} \mathrm{CHEN}^{2},{\text { YANMING } \mathrm{LI}^{3} \text { and JI-FU WEI }}^{2}$ \\ ${ }^{1}$ Department of Geriatrics, Beijing Hospital, National Center of Gerontology, Beijing 100730; \\ ${ }^{2}$ Research Division of Clinical Pharmacology, The First Affiliated Hospital of Nanjing Medical University, \\ Nanjing, Jiangsu 210029; ${ }^{3}$ Department of Respiratory and Critical Care Medicine, Beijing Hospital, \\ National Clinical Research Center for Respiratory Disease, Beijing 100730, P.R. China
}

Received December 29, 2015; Accepted October 21, 2016

DOI: $10.3892 /$ ijmm.2016.2790

\begin{abstract}
Cockroach (CR) allergies caused by the American cockroach hyave been recognized to be repsonsible for IgE-mediated type I hypersensitivity worldwide. Per a 10 is one of the recognized main allergens of the American CR. In a previous study, we examined another American CR allergen, Per a 9 in patients with CR allergies and examined epitope sequences in this allergen. In the present study, we aimed to examine epitope sequences in the Per a 10 allergen. for this purpose, the Per a 10 gene was cloned and expressed in Escherichia coli (E. coli) systems. Our results revealed that 9 out of $16(56.3 \%)$ sera from patients with American CR allergies reacted to Per a10, as assessed by ELISA, confirming that Per a 10 is a major allergen of the American CR. Our results also revealed that the expression of CD63 and CCR3 on passively sensitized basophils (obtained sera of patients with American CR allergies) was increased by approximately 2.3 -fold, indicating that recombinant Per a 10 is functionally active. In addition, 3 immunoinformatics tools, namely the DNAStar Protean system, the Bioinformatics Predicted Antigenic Peptides (BPAP) system and the BepiPred 1.0 server were used to predict the peptides and the results revealed 8 peptides (2-12, 55-67, 98-120, 125-133, 149-160, 170-182, 201-208 and 223-227) as potential B cell epitopes of the Per a 10 allergen. Moreover, Per a 10 was predicted to have $3 \mathrm{~T}$ cell epitope sequences,
\end{abstract}

Correspondence to: Dr Yanming Li, Department of Respiratory and Critical Care Medicine, Beijing Hospital, National Clinical Research Center for Respiratory Disease, 1 Dahua Road, Beijing 100730, P.R. China

E-mail: lymyl@263.net

Professor Ji-Fu Wei, Research Division of Clinical Pharmacology, The First Affiliated Hospital, Nanjing Medical University, 300 Guangzhou Road, Nanjing, Jiangsu 210029, P.R. China

E-mail: weijifu@hotmail.com

*Contributed equally

Key words: cockroach, allergen, Per a 10, epitopes, Escherichia coli, $\operatorname{IgE}$ namely $83-92,139-147$ and 162-170. The findings of our study on the CR allergen may prove to be useful in the development of peptide-based vaccine for the prevention and/or treatment of CR allergies.

\section{Introduction}

A number of individuals worlwide are known to suffer from cockroach (CR) allergies and the $\mathrm{CR}$ allergies are considered to play an important role in IgE-mediated type I hypersensitivity since 1964 (1). German CR [Blattella germanica (Bla g)], American CR [Periplaneta Americana (Per a)] and smoky brown CR (Periplaneta fuliginosa) are the main species which are known to cause allergies worldwide (2). In China, a number of patients with allergies are found to positive for CR allergens in the skin prick test (SPT); $25.7 \%$ are shown to be positive for the American CR and $18.7 \%$ are shown to be positive for the German CR (3).

The adult American CR has $22 \mathrm{IgE}$ binding components with pooled sera from patients, including the proteins of 23,28 , 35, 38, 40, 49, 72, 78 and $97 \mathrm{kDa}$ as major allergens (4). Some of these allergens namely, Per a 1 (5), Per a 2 (previously known as Cr PI) (6), Per a 3 (7), Per a 4 (8), Per a 5 (9), Per a 6 (10), Per a 7 (11), Per a 9 (previously known as Periplaneta americana arginine kinase) (12,13), Per a 10 (14), Per a 11 (15) and Per a 12 (15) have been characterized. Among these allergens, Per a 10 was isolated from American CR extract using a benzamidine sepharose column and characterized by immunobiochemical methods. It is a serine proteinase with a molecular weight of approximately $28 \mathrm{kDa}(14)$. Per a 10 was recognized as a major allergen, showing $\operatorname{IgE}$ reactivity with $>80 \%$ of cockroach sensitized patients by skin tests and immunoblot (14). Further studies found that Per a 10 can modulate CD40 expression on the dendritic cell surface through the nuclear factor $-\kappa \mathrm{B}(\mathrm{NF}-\kappa \mathrm{B})$ pathway (16) and can promote the dendritic cell type 2 phenotype via the upregulation of CD86, increased high interleukin-6 (IL-6) secretion and reduced IL-12 secretions (17).

Recent studies have suggested that CR immunotherapy may be a promising treatment strategy with immunomodulatory and clinical effects $(18,19)$. However, patients with CR allergies often exhibit complex sensitization patterns to multiple 
CR-associated proteins and an immunodominant allergen has not yet been identified (20). To realize the full potential of this treatment modality, further investigations of CR allergens are warranted. The identification of B cell epitopes (IgE-binding epitopes) of an allergen is helpful in designing sequences for more accurate and safer peptide-based allergen diagnosis and immunotherapeutic agents. The IgE-binding epitopes of Per a 1 (21), Per a 2 (22), Per a 3 (23), Per a 4 (8) and Per a 6 (10) have previously been identified by experimental or bioinformatics methods. T cell epitopes have been successfully identified based on computer simulation over the past decade. Extracellular peptides have to bind to major histocompatibility complex (MHC) class II to stimulate T lymphocyte responses. Thus, $\mathrm{T}$ cell epitopes have been predicted indirectly by the identification of MHC-binding molecules (20). In a previous study (13), using in silico analysis, we performed epitope prediction and functional analysis of the Per a 9 allergen from the American CR. In the present study, in continuation of our investigation on Amiercan CR allerens, we firstly cloned, expressed the American CR major allergen Per a 10 and identified the B and T cell epitopes of the Per a 10 allergen using an in silico approach. Our findings suggest the potential utility of these allergens and epitopes in the development of peptide-based vaccines for the prevention and/or treatment of CR allergies.

\section{Materials and methods}

Patients and samples. As in our previous study (13), in this study, 16 patients with allergic rhinitis who were found to have positive SPT results and positive results for the serum IgE test for the American CR extract (by using ImmunoCAP assay; Pharmacia Diagnostics AB, Uppsala, Sweden) and 6 healthy controls (HC) who showed negative results for the serum IgE test were recruited. Blood samples were obtained from all participants after obtaining written informed consent and this study was approved by the Ethics Committee of the First Affiliated Hospital of Nanjing Medical University, Nanjing, China.

Molecular cloning of the American CR Per a 10 gene. Total RNA was isolated from female CRs using TRIzol reagent (Invitrogen, Carlsbad, CA, USA). Total RNA was quantified by measuring the absorbance ratios at 260/280 nm. Total RNA was reverse transcribed into cDNA using oligo(dT) by reverse transcriptase using a commercial cDNA synthesis kit according to the manufacturer's instructions (Takara Biotech Co., Dalian, China). The gene encoding Per a 10 was amplified by PCR using primers based on the nucleotides sequence of the Per a 10 gene (AY792954.1; forward, 5'-ATGC TTCGCTACCTGGTACTT-3' and reverse, 5'-TTAGTTGA CTCCAGTCTGTTC-3'). The PCR conditions were as follows: $98^{\circ} \mathrm{C} / 5 \mathrm{~min}(1 \mathrm{cycle}), 98^{\circ} \mathrm{C} / 10 \mathrm{sec}, 50^{\circ} \mathrm{C} / 15 \mathrm{sec}$ and $72^{\circ} \mathrm{C} / 1 \mathrm{~min}$ (35 cycles), and $72^{\circ} \mathrm{C} / 5$ min ( 1 cycle). The purified PCR product was cloned into the pMD18-T vector (Takara Biotech Co.) and transformed into the Escherichia coli (E. coli) strain DH5 $\alpha$. The inserts were confirmed by DNA sequencing.

Expression and purification of Per a 10 in E. coli. As previously described with some modifications (13), the Per a 10 gene was subcloned into the pET-22b(+) vector (Novagen, Madison, WI, USA) using the NdeI and XhoI sites and confirmed by DNA sequencing. The recombinant pET22b(+)-Per a 10 plasmid was transformed into the Rosette-gami E. coli host strain. The selected pET22b(+)-Per a 10-transformed Rosette-gami E. coli was inoculated into $5 \mathrm{ml}$ of LB-ampicillin broth, and incubated at $37^{\circ} \mathrm{C}$ overnight. Five milliliters of the culture were inoculated into $500 \mathrm{ml}$ of fresh LB-ampicillin broth and incubated at $28^{\circ} \mathrm{C}$ while shaking at $200 \mathrm{rpm}$ until the optical density (OD) at $A_{600 \mathrm{~nm}}$ reached 0.5 . Isopropyl- $\beta$-D-thiogalactopyranoside (IPTG) was added to a final concentration of $1 \mathrm{mM}$ and the culture was incubated for a further $7 \mathrm{~h}$. The bacterial cells were harvested by centrifugation at $4,000 \mathrm{xg}$ at $4^{\circ} \mathrm{C}$ for $20 \mathrm{~min}$, and were lysed in lysis buffer by sonication at $20 \mathrm{kHz}, 2 \mathrm{~min}$ pulse-on, $3 \mathrm{~min}$ pulse-off. The recombinant Per a 10 was mainly contained in inclusion bodies. The inclusion bodies were collected by centrifugation at $12,000 \mathrm{xg}$ at $4^{\circ} \mathrm{C}$ for $20 \mathrm{~min}$. Following solubilization of the inclusion bodies by $6 \mathrm{M}$ urea, the supernatant was loaded onto a Nickel column (Genscript, Nanjing, China), washed with running buffer containing $50 \mathrm{mM}$ Tris- $\mathrm{HCl}, 300 \mathrm{mM} \mathrm{NaCl}$ (pH 8.0), and eluted with elution buffer containing $50 \mathrm{mM}$ Tris- $\mathrm{HCl}, 300 \mathrm{mM} \mathrm{NaCl}, 250 \mathrm{mM}$ imidazole. The eluted fractions were collected and dialyzed with 6-4-2-1-0.5-0 M urea, each for $2 \mathrm{~h}$.

Immunoreactivity of recombinant Per a 10 with serum from patients with $C R$ allergies. A 96-well plate was coated with recombinant $100 \mu \mathrm{l}$ Per a $10(10 \mu \mathrm{g} / \mathrm{ml})$ in carbonate-bicarbonate buffer $(0.05 \mathrm{M}, \mathrm{pH} 9.6)$ overnight at $4^{\circ} \mathrm{C}$. Human serum samples (1:20 dilution in PBS-Tween-20 with 2\% BSA) were then added to the plates and incubated for $2 \mathrm{~h}$ at room temperature. Following IgE binding, the plates were incubated with horseradish peroxidase-labeled goat anti-human $\operatorname{IgE}(1: 2,500$ dilution) (KPL, Inc., Gaithersburg, MD, USA), and the color was developed with tetramethylbenzidine (TMB) peroxidase substrate. The plates were read on a microplate reader (Eon; BioTek, Winooski, Vermont, USA) at an absorbance of $405 \mathrm{~nm}$. The cut-off value of the enzyme-linked immunosorbent assay (ELISA) was calculated as the mean of the negative controls plus 2 SDs, as previously described (9).

Immunoblot analysis of the IgE reactivity of Per a 10. Immunoblots for the detection of $\operatorname{IgE}$ reactivity of Per a 10 were performed as described previously $(24,25)$. Per a $10(5 \mu \mathrm{g})$ was conducted to a sodium dodecyl sulfate-polyacrylamide gel electrophoresis (SDS-PAGE; gel concentration of 15\%) under reduced conditions and then transferred onto nitrocellulose membranes. The nitrocellulose membranes were firstly incubated with sera from patients with American CR allergies (1:5 to $1: 20$ in PBS-Tween-20 with $1 \%$ BSA, $10 \%$ normal goat serum) for $90 \mathrm{~min}$, and then incubated with peroxidase-labeled anti-human IgE monoclonal antibody (074-1004, KPL, Inc., Gaithersburg, MD, USA). The positive protein bands were visualized by incubating the membranes with TMB peroxidase substrate. Sera from 2 non-allergic healthy subjects were used as the negative controls.

Basophil activation test. The expression of CD63 and CCR3 on the basophil surface is considered as an indicator of basophil activation (26,27). Briefly, and as previously described (13), 
peripheral blood mononucleated cells (PBMCs) from 4 healthy volunteers were separated by Ficoll-Paque density gradient, and treated with $10 \mathrm{ml} \mathrm{LS}$ solution $(1.3 \mathrm{M} \mathrm{NaCl}, 0.005 \mathrm{M}$ $\mathrm{KCl}$ and 0.01 lactic acid, $\mathrm{pH} 3.9$ ) for $2 \mathrm{~min}$ at $8^{\circ} \mathrm{C}$. Following neutralization with $12 \%$ Tris $(\mathrm{pH} 10.9)$, non-specific IgE on basophils was stripped off. Subsequently, the basophils were passively sensitized with the sera of patients with American CR allergies or from the healthy non-allergic healthy controls $(n=4$, 1 in 10 dilution, $2 \mathrm{~h}$ at $37^{\circ} \mathrm{C}$ ) (same patients and controls as described above) as previously described (23). The sensitized basophils were challenged with Per a $10(1.0 \mu \mathrm{g} / \mathrm{ml})$ for $15 \mathrm{~min}$ at $37^{\circ} \mathrm{C}$. CCR3-PE-labelled antibody (85-12-1939-42; eBioscience Inc., San Diego, CA, USA) and anti-human CD63-FITC antibody (HH-MHCD63014; Invitrogen, Camarillo, CA, USA) were added to cells for $15 \mathrm{~min}$ at $37^{\circ} \mathrm{C}$. Flow cytometric analysis of CD63 and CCR3 was performed at $488 \mathrm{~nm}$ on a FACSAria flow cytometer (Becton-Dickinson, Franklin Lakes, NJ, USA) and analyzed by FACSDiva software.

Sequence retrieval Per a 10 homolog and phylogenetic analysis. The complete amino acid sequence of Per a 10 was used as query to search for homologous sequences by tBLASTn in NCBI (blast.ncbi.nlm.nih.gov/Blast.cgi) (10,28-30). The phylogenetic tree of Per a 10 and its homolog was obtained by the maximum-likelihood (ML) method on the basis of the JTT amino acid sequence distance implemented in MEGA 5.1, the reliability was evaluated by the bootstrap method with 1,000 replications (28-32).

Physiochemical analysis and post-translational patterns and motifs of Per a 10. As previously described (13), physiochemical analysis, including molecular weight, theoretical pI, amino acid composition, instability index, aliphatic index and the grand average of hydropathicity (GRAVY) of Per a 10 was performed using the ProtParam tool (http://web.expasy.org/ protparam/) (33).

Secondary structure prediction of Per a 10. As previously described (13), the secondary structure of Per a 10 was assessed by PSIPRED (bioinf.cs.ucl.ac.uk/psipred) (34) and NetSurfP ver. 1.1 (www.cbs.dtu.dk) (35).

Homology modeling and validation. As previously described (13), the best homologous templates for Per a 10 were selected using the PSI-BLAST server (http://blast.ncbi. nlm.nih.gov/Blast.cgi) in NCBI and Swiss-model server (http:// swissmodel.expasy.org/) and used for homology modeling. The modeled protein structure of Per a 10 was built by SWISSMODEL (http://swissmodel.expasy.org/). An initial structural model was generated and checked for recognition of errors in 3D structure by PROCHECK (36), ERRAT (verification of protein structures: patterns of nonbonded atomic interactions) $(33,37)$ and VERIFY_3D (a method to identify protein sequences that fold into a known three-dimensional structure. Assessment of protein models with 3-dimensional profiles) programs in Structural Analysis and Verification Server (http:// nihserver.mbi.ucla.edu/SAVES/) $(33,37)$.

In silico prediction of B cell epitopes of Per a 10. As previously described (13), 3 immunoinformatics tools, namely the
DNAStar Protean system (38), bioinformatics predicted antigenic peptides (BPAP) system (http://imed.med.ucm.es/Tools/ antigenic.pl) and the BepiPred 1.0 server (http://www.cbs.dtu. $\mathrm{dk} /$ services/BepiPred/) were used to predict the B cell epitopes of Per a $10(39,40)$. The ultimate consensus epitope results were obtained by combining the results of the 3 tools (41). If the results of all 3 methods were non-epitope, the consensus result was then considered $0 \%$ epitope. Similarly, if the predicted results had only one or no non-epitope, the consensus result was considered 67 or $100 \%$ epitope, respectively. Finally, the regions whose consensus epitope result was 67 or $100 \%$ were chosen as the final potential epitope regions.

In silico prediction of $T$ cell epitopes. As previously described (13), for HLA-DR-based T cell epitope prediction, the artificial neural network-based alignment (NN-align) method NetMHCIIpan-3.0 (http://www.cbs.dtu.dk/services/ NetMHCIIpan/) was applied (42). We used HLA-DR 101, HLA-DR 301, HLA-DR 401 and HLA-DR 501. The ultimate HLA-DR-based Tcellepitoperesultswereobtainedbycombining those 4 results together that if 3 of them showed epitope, and then the consensus result was epitope. For HLA-DQ alleles, NetMHCII-2.2 (http://www.cbs.dtu.dk/services/NetMHCII/) was used (43). We only used HLA-DQA10501-DQB10201, HLA-DQA10301-DQB10302, HLA-DQA10401-DQB10402, and HLA-DQA10102-DQB10602. As a result, the ultimate consensus epitope results were obtained by combining the results of the HLA-DR-based T cell epitope and HLA-DQ-based T cell epitope. B cell and T cell epitopes identified by computational tools were mapped onto linear sequence and on the three dimensional model of Per a 10 to determine their position and secondary structure elements involved.

Statistical analysis. Data are expressed as the means \pm SE for the indicated number of independently performed duplicated experiments. Statistical significance between means was analyzed by one-way ANOVA or the Student's t-test utilizing the SPSS 13.0 version. A valoue of $\mathrm{P}<0.05$ was considered to indicate a statistically significant difference.

\section{Results}

Molecular cloning of the Per a 10 allergen of the American $C R$. The cDNA encoding the Per a 10 gene was amplified by PCR. It is 771 bp gene and encodes a 256 amino acids protein. The sequence homology with the published one (Accession no. AY792954.1) was $100 \%(256 / 256)$ at the protein level. Among the 256 amino acid protein, MLRYLVLASLIACSLS is a signal peptide, and AVPKAKRPRLDGR is a pro-peptide, which would be removed in the mature Per a 10 . So the mature Per a 10 contained 227 amino acids.

Expression and purification of Per a 10 in E. coli. The American CR allergen Per a 10 was subcloned into the pET-22b(+) vector and transformed into the Rosette-gami E. coli host strain. Per a 10 was firstly expressed at temperatures of $16^{\circ} \mathrm{C}, 28^{\circ} \mathrm{C}$ or $37^{\circ} \mathrm{C}$ (Fig. 1A). It was found that Per a 10 was expressed mainly in the inclusion body and $28^{\circ} \mathrm{C}$ was the optimal temperature condition for its expression. Thus, the condition of $1 \mathrm{mM}$ IPTG and a temperature of $28^{\circ} \mathrm{C}$ was 


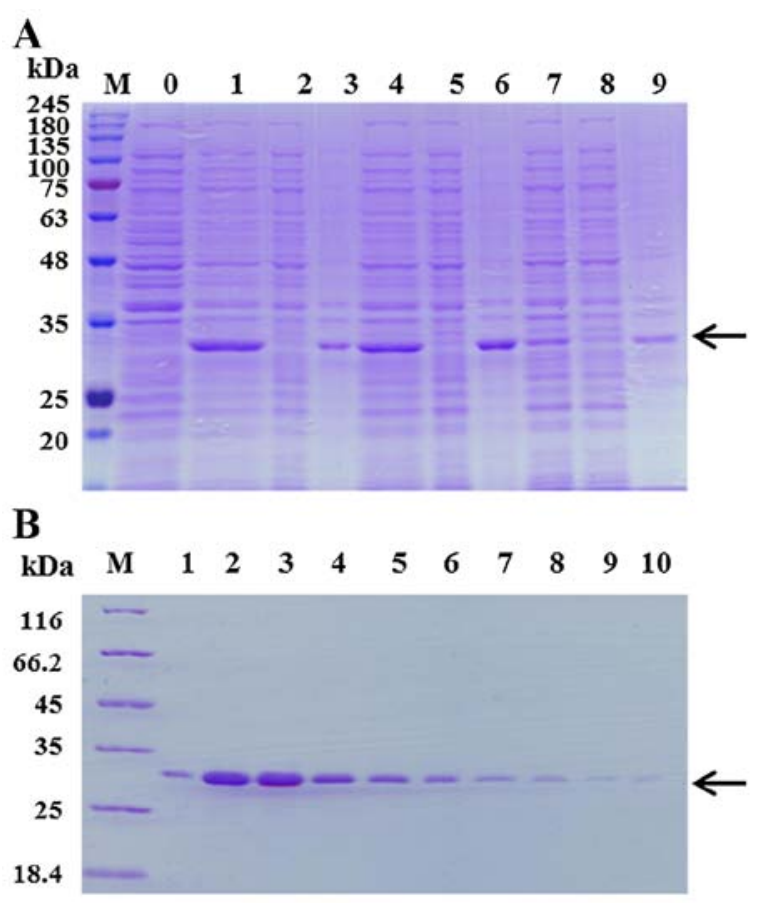

Figure 1.Expression and purification of Per a 10 in E.coli.(A) Per a 10 expressed at $16^{\circ} \mathrm{C}, 28^{\circ} \mathrm{C}$ or $37^{\circ} \mathrm{C}$ was analyzed by sodium dodecyl sulfate-polyacrylamide gel electrophoresis (SDS-PAGE). Lane M, protein makers; lane 1, the total protein of un-induced bacteria; lane 2 , the total protein of bacteria induced by $1 \mathrm{mM}$ isopropyl- $\beta$-D-thiogalactopyranoside (IPTG) at $37^{\circ} \mathrm{C}$; lane 3 , the supernatant of the bacteria induced by $1 \mathrm{mM} \mathrm{IPTG}$ at $37^{\circ} \mathrm{C}$; lane 4 , the precipitant of the bacteria induced by $1 \mathrm{mM} \mathrm{IPTG}$ at $37^{\circ} \mathrm{C}$; lane 5 , the total protein of bacteria induced by $1 \mathrm{mM} \mathrm{IPTG}$ at $28^{\circ} \mathrm{C}$; lane 6 , the supernatant of the bacterial induced by $1 \mathrm{mM} \mathrm{IPTG}$ at $28^{\circ} \mathrm{C}$; lane 7 , the precipitant of the bacterial induced by $1 \mathrm{mM}$ IPTG at $28^{\circ} \mathrm{C}$; lane 8 , the total protein of bacteria induced by $1 \mathrm{mM}$ IPTG at $16^{\circ} \mathrm{C}$; lane 9 , the supernatant of the bacteria induced by $1 \mathrm{mM} \mathrm{IPTG}$ at $16^{\circ} \mathrm{C}$; lane 10 , the precipitant of the bacteria induced by $1 \mathrm{mM}$ IPTG at $16^{\circ} \mathrm{C}$. The arrow represents Per a 10 protein. (B) The purification of Per a 10 expressed in E. coli. Lane M, protein makers; lanes 1-10, washing with $250 \mathrm{mM}$ imidazole. The arrow represents Per a 10 protein.

selected for the large expression and purification of Per a 10 . The dissolved Per a 10 inclusion body was purified by a $\mathrm{Ni}$ column. After the successful renaturation of purified Per a 10, approximately $1.4 \mathrm{mg}$ recombinant Per a 10 was obtained from $500 \mathrm{ml}$ of cell culture. The purity of the purified Per a 10 was identified by SDS-PAGE. It showed a single band with an apparent molecular weight of $30 \mathrm{kDa}$ (Fig. 1B).

Immuno-reactivity of Per a 10 to $\mathrm{IgE}$. In order to determine the allergenicity of Per a 10, the ability of Per a 10 to bind $\operatorname{IgE}$ in sera from patients with American CR allergies was determined by a direct ELISA technique. The sera from patients 1, 3, 5, 6, 8 , $9,11,14$ and 15 showed positive IgE reactivity to Per a 10 . The results revealed that 9 out of $16(56.3 \%)$ of the sera from these patients reacted to Per a 10 (Fig. 2A). The IgE binding activity of Per a 10 in a representative group of 3 patients and 2 healthy controls was assessed by western blot analysis and the results are illustrated in Fig. 2B.

Per a 10 induces human basophil activation. Per a 10 at $1.0 \mu \mathrm{g} /$ $\mathrm{ml}$ induced an approximately 2.3 -fold increase in the number of CD63 and CCR3 double-positive cells following incubation

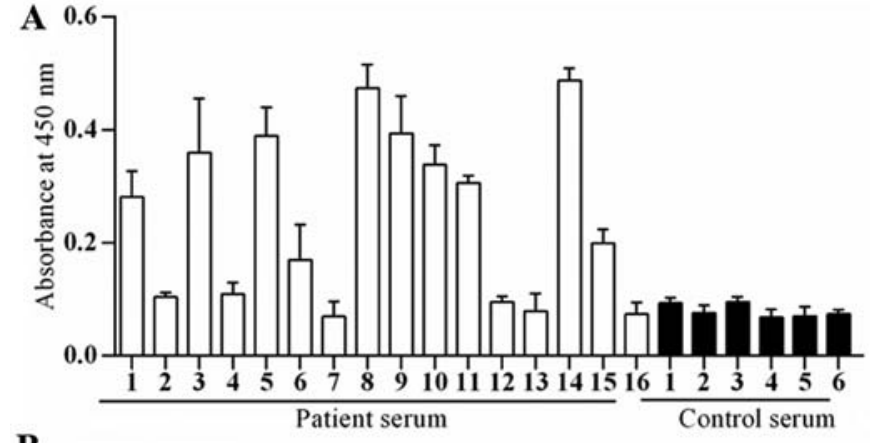

B

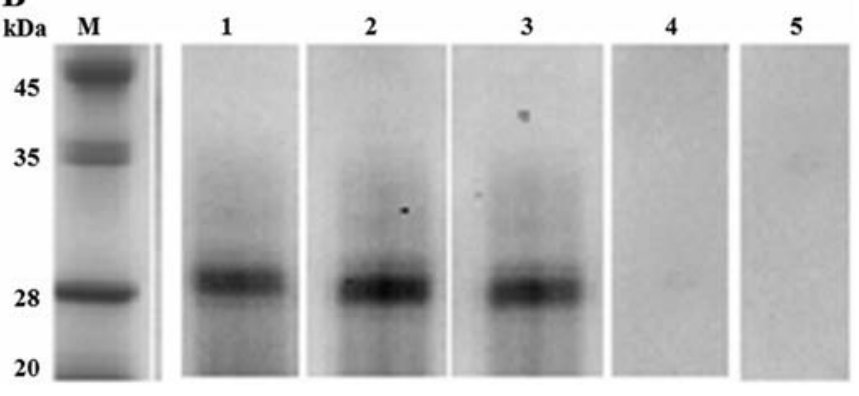

Figure 2. (A) Analysis of specific IgE reactivity of recombinant Per a 10 by direct ELISA. Sera were collected from patients with American cockroach (CR) allergies and healthy control subjects. The values shown are the mean \pm SDs from triplicate experiments. (B) Western blot analysis of $\mathrm{IgE}$ reactivity to Per a 10 sera from patients with American CR allergies. Lanes 1-3, Per a 10 reacted with the serum from patients 3,8 and 14 , respectively; lanes $4-5$, Per a 10 reacted with serum from control subjects 1 and 2 , respectively.

with passively sensitized basophils from the sera of patients with American CR allergies. We found that Per a 10 had no effect on the basophils sensitized by the sera from the healthy controls (Fig. 3). These results are similar to those of our previous study on the Per a 9 American CR allergen, where we had also found that thye basophils sensitized by the sera from the healthy controls were not affected, whereas the basophils sensitized by sera from the allergic patients exhibited a 4.2 -fold increase in the number of CD63 and CCR3 double-positive cells (13).

Sequence retrieval and phylogenetic analysis. Uniprot and tBLASTn were used to search the homologous sequences of Per a 10. As a result, 20 sequences were obtained. Moreover, the results of phylogenetic analysis of Per a 10 and its homologous sequences inferred by the ML method are shown in Fig. 4.

Physiochemical analyses of Per a 10. The primary structure of Per a 10 contained 227 amino acids and the molecular weight was 23295.6. The theoretical pI was 4.28 and the aliphatic index was 76.04 . The GRAVY was 0.001 , indicting that Per a 10 exhibited a hydrophilic character. The instability index was $34.61(<40)$, indicating that the Per a 10 protein was stable.

Structural analysis of Per a 10. Secondary structure prediction with PSIPRED identified $2 \alpha$-helices and $14 \beta$-sheets in Per a 10. PredictProtein predicted $2 \alpha$-helices and $12 \beta$-sheets. Moreover, NetSurfP v1.1 also predicted $2 \alpha$-helices and $12 \beta$-sheets. In total, all secondary structure prediction yielded the same results: the secondary structure of Per a 10 contained $2 \alpha$-helices and $14 \beta$-sheets and the results are presented in Table I. 
Table I. The predicated secondary structure of Per a 10.

Secondary structural prediction methods

$\alpha$-helices

$\beta$-sheets

\begin{tabular}{|c|c|c|}
\hline PSIPRED & $147-153,217-225$ & $\begin{array}{l}5-6,15-19,24-32,35-39,51-55,58-60,65-74,86-92,105-106,117-122, \\
138-145,184-187,190-196,208-212\end{array}$ \\
\hline NetSurfP ver1.1 & $147-153,213-225$ & $\begin{array}{l}14-20,24-30,35-42,50-59,65-74,87-92,102-106,117-122,138-145, \\
165-167,190-196,209-212\end{array}$ \\
\hline PredictProtein & $148-151,213-224$ & $\begin{array}{l}14-20,24-32,35-43,51-59,65-79,87-92,101-106,117-122,137-146, \\
162-168,185-197,209-212\end{array}$ \\
\hline Overall results & $147-153,217-225$ & $\begin{array}{l}14-20,24-32,35-39,51-55,58-60,65-74,87-92,117-122,137-145,184-187, \\
185-196,208-212 .\end{array}$ \\
\hline
\end{tabular}

A

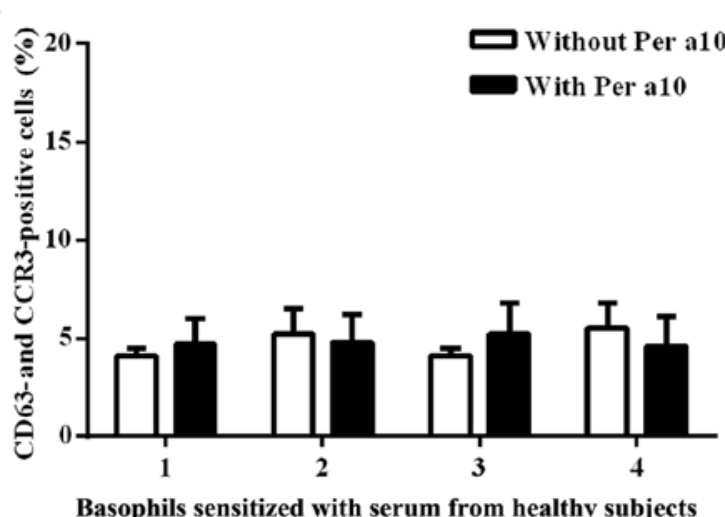

B

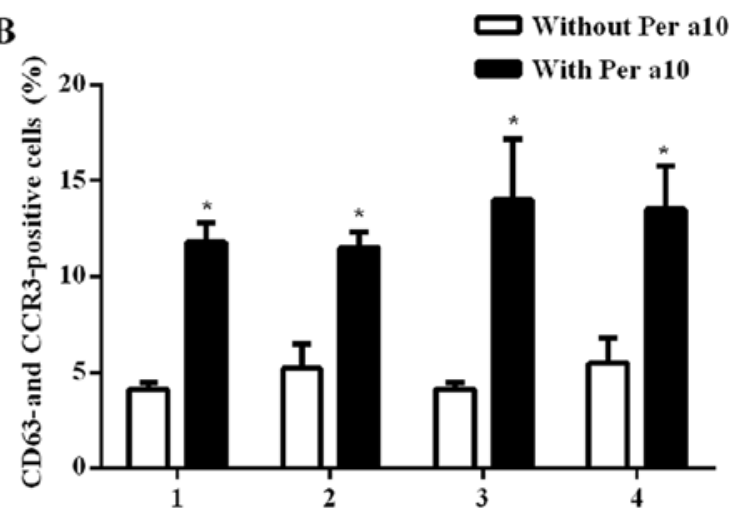

Basophils sensitized with serum from patients with CR allergies

Figure 3. Induction of basophil activation by recombinant Per a 10. Following non-specific IgEs on basophils being stripped off, cells from each donor were passively sensitized with sera from (A) 4 different healthy subjects or (B) from 4 different patients with American cockroach (CR) allergies, and were then challenged with Per a 10 at $1.0 \mu \mathrm{g} / \mathrm{ml}$. The values shown are the means $\pm \mathrm{SE}$ for the sera from 4 different subjects. $\mathrm{P}<0.05$ in comparison with the corresponding carrier alone control.

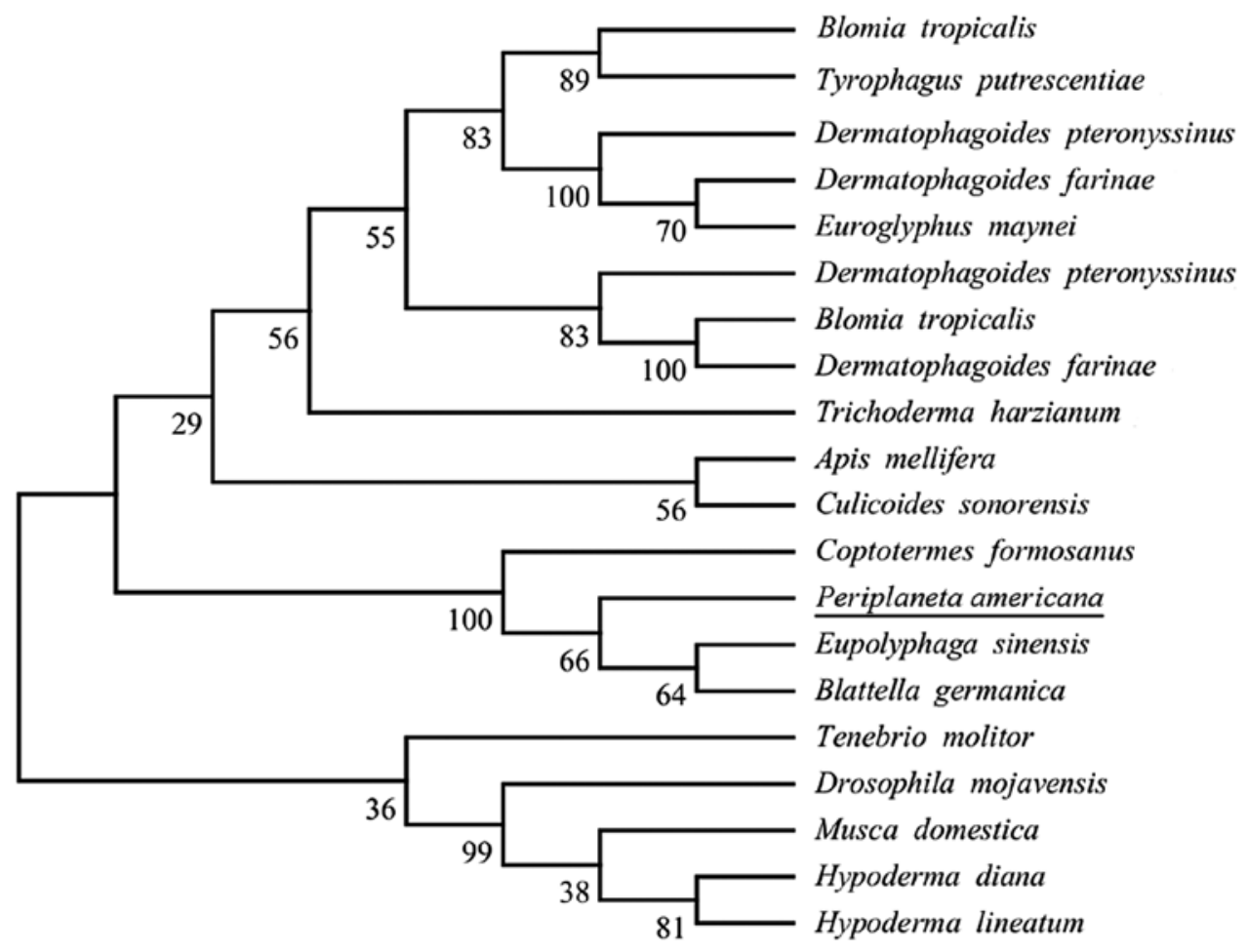

Figure 4. Phylogenetic relationship of Per a 10 allergen amino acid sequence with other homologs. 
A
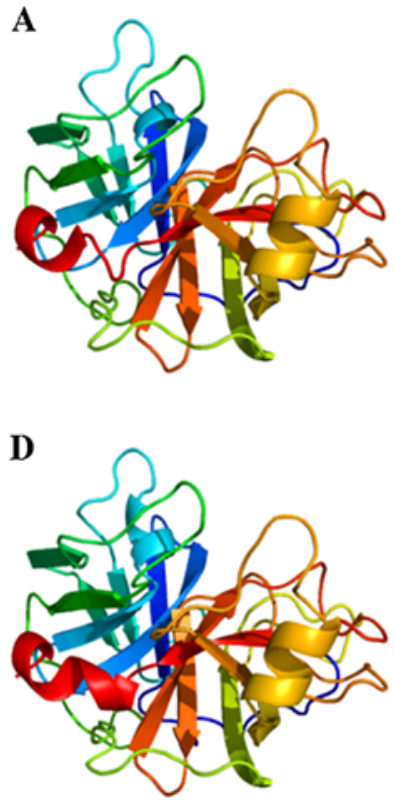
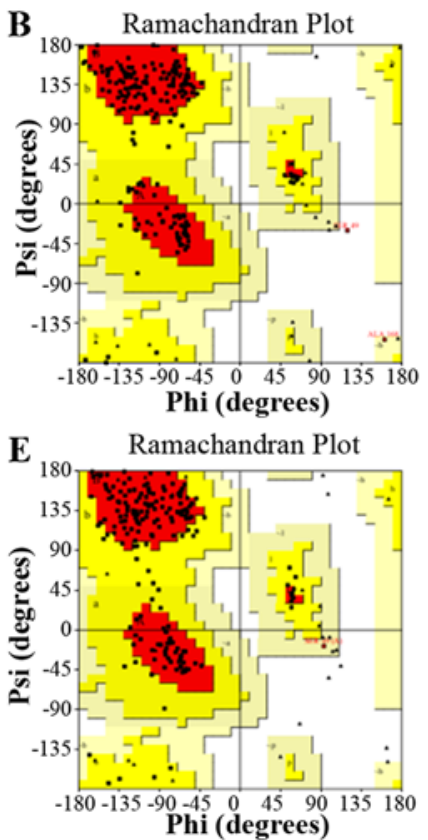

C Chain\#:1 1 quality factor**:85.167

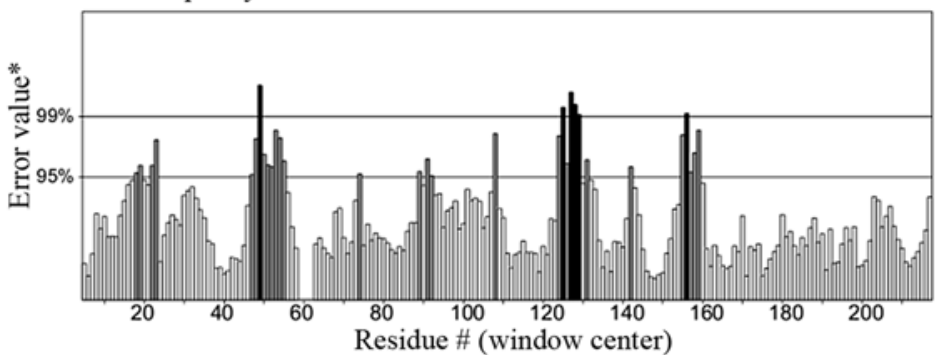

F Chain\#:1 1 (quality factor $*: 86.408$

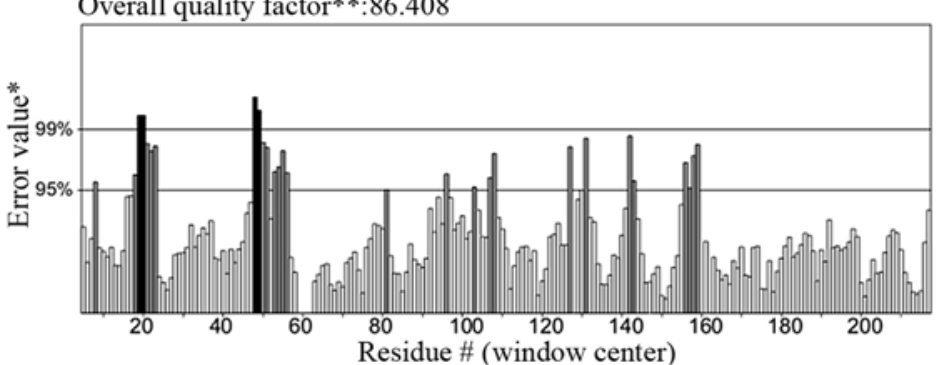

Figure 5. Three-dimensional structure and validation of Per a 10 homology model. (A) The preliminary protein structure of Per a 10 homology model. (B) Ramachandran plot of the preliminary protein structure of Per a 10. (C) Validation of the preliminary protein structure of Per a 10 by the ERRAT program. (D) The optimized protein structure of Per a 10 homology model. (E) Ramachandran plot of the optimized protein structure of Per a 10. The residures in most favored regions, additional allowed regions, generously allowed regions and disallowed regions are shown in red, yellow, grey and white in the Ramachandran plot, respectively. (F) Validation of the optimized protein structure of Per a 10 by the ERRAT program.

Homology modeling and validation of Per a 10. The search for the proteins Per a 10 with a known tertiary structure in the PDB yielded pacific salmon anionic Trypsin (PDB Accession no. 1MBQ; https://www.ncbi.nlm.nih.gov/Structure/mmdb/ mmdbsrv.cgi?uid=1MBQ) showing the highest sequence identity (44\% with Per a 10$)$. As a result, the $1 \mathrm{MBQ}$ template was used for homology modeling. The tertiary structure of Per a 10 constructed by the homology model is shown in Fig. 5A. As indicated by the Ramachandran plot (Fig. 5B), $81.9 \%$ of the residues in the model structure were within the most favored regions, $17.5 \%$ of the residues were in the allowed region, and $0.6 \%$ of the residues were in the disallowed region. As indicated by the ERRAT program, the results (Fig. 5C) revealed that the overall quality factor was 85.167 , which indicated that the structure had a relatively high resolution. As indicated by the VERIFY_3D program, the results revealed that $90.54 \%$ of the residues had an average 3D (atomic model)-1D (amino acid sequence) score of $>0.2$, which is not sufficient. Thus, we optimized this modeled structure by Chiron (http://troll.med. unc.edu/chiron/login.php). The optimized Per a 10 structure is shown in Fig. 6D. The results of the Ramachandran plot revealed that $79.7 \%$ of the residues in the model structure were within the most favored regions, $20.3 \%$ of the residues were in the allowed region, and $0 \%$ of the residues were in the disallowed region (Fig. 5D). As indicated by the ERRAT program, the result (Fig. 5E) showed that the overall quality factor is 86.408 which mean the structure has a relatively high resolution. As indicated by the VERIFY_3D program (Fig. 5F), the results indicated that $94.14 \%$ of the residues had an average 3D (atomic model)-1D (amino acid sequence) score of $>0.2$, and this results was favourable. Based on these validations, the optimized Per a 10 structure was adopted for further analysis.
B cell epitope prediction of Per a 10. Bases on surface accessibility, fragment flexibility and hydrophobicity analysis of Per a 10 , the final predicting regions of Per a 10 by DNAstar were obtained as: 2-10, 55-65, 109-115, 125-133, 147-160, 170-182, 201-208 and 223-227. The predicted results of the BPAP system were 8-20, 22-51, 63-74, 78-96, 98-110, 113-120, $132-147,149-156,162-168,182-203$ and 205-218. The predicted results of the BepiPred 1.0 server were 1-12, 46-67, 74-80, 95-134, 149-160, 169-186, 201-211 and 223-227. The ultimate results of the 3 immunoinformatics tools finally predicted 8 peptides (2-12, 55-67, 98-120, 125-133, 149-160, 170-182, 201-208 and 223-227) and these peptides are shown in Fig. 6.

$T$ cell epitope prediction. For HLA-DR-based T cell epitope prediction of Per a 10 , the final predicting regions of HLA-DR 101, HLA-DR 301, HLA-DR 401 and HLA-DR 501 are shown in Table II and the ultimate results of HLA-DR-based $\mathrm{T}$ cell epitope prediction finally predicted 2 peptides (83-91 and 139-147). For HLA-DQ alleles, the final results of HLA-DQA 10101-DQB10501, HLA-DQA10301-DQB10302, HLA-DQA10401-DQB10402, and HLA-DQA10102-DQB10602 are also shown in Table II and the ultimate results of these 4 methods finally predicted 2 peptides, 84-92 and 162-170. As a result, Per a 10 was predicted to have $3 \mathrm{~T}$ cell epitope sequences, namely 83-92 (a combination of peptide 83-91 and 84-92), 139-147 and 162-170 as shown in Fig. 6.

\section{Discussion}

To better understand the Per a 10-mediated CR allergies and with an aim improve the diagnosis and treatment of CR allergies, 
Table II. The T cell epitope prediction of Per a 10.

$\begin{array}{ll}\text { HLA types } & \text { Location of the prediction results }\end{array}$

\section{HLA-DR101}

HLA-DR301

HLA-DR401

HLA-DR501

HLA-DQA10101-DQB10501

HLA-DQA10501-DQB10201

HLA-DQA10301-DQB10302

HLA-DQA10401-DQB10402

HLA-DQA10102-DQB10602

The final predicted $\mathrm{T}$ cell epitopes
$1-9,14-22,17-25,18-26,20-28,22-30,24-32,25-33,26-34,28-36,31-39,35-43,42-50$, 43-51, 45-53, 53-61, 65-73, 69-77, 70-78, 72-80, 80-88, 81-89, 85-93, 87-95, 89-97, 96-104, 99-107, 102-110, 105-113, 107-115, 117-125, 136-144, 137-145, 184-192, 186-194, 187-195, 191-199, 206-214, 209-217, 210-218, 213-221

8-16, 72-80, 81-89

83-91, 87-105, 89-97, 136-144, 139-147, 184-192, 185-193

18-26, 35-43, 46-54, 52-60, 83-91, 85-93, 89-97, 139-147, 209-217, 210-218

78-86, 81-89, 184-202

$13-21,38-46,78-86,80-88,157-165$

82-90, 84-92, 90-98, 162-170

11-19, 84-92, 162-170, 195-203

20-28, 25-33, 26-34, 31-39, 34-42, 37-45, 43-51, 47-55, 61-69, 66-74, 67-75, 84-92, $87-95,90-98,97-105,102-110,132-140,162-170,165-173,190-198,217-225$

$83-92,139-147,162-170$
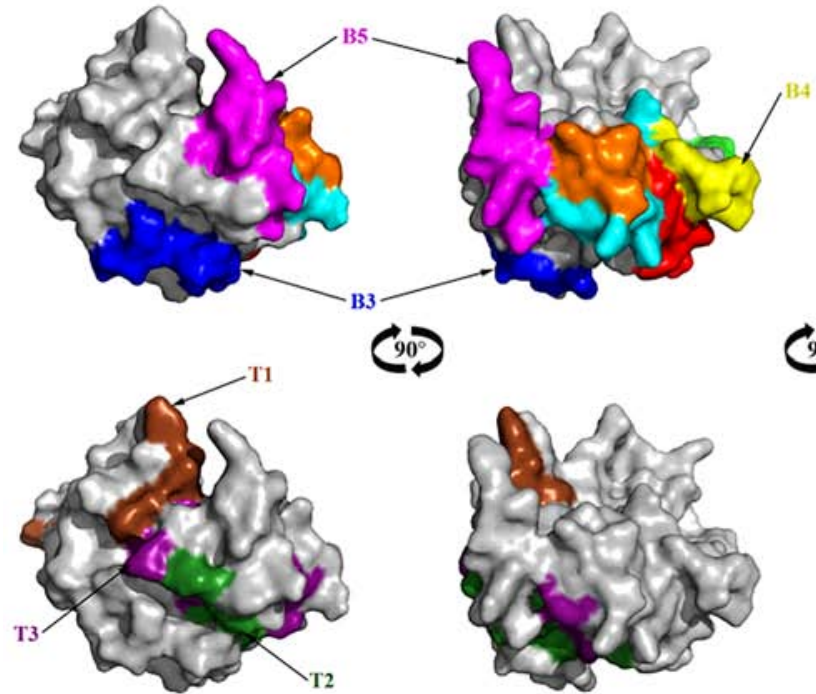
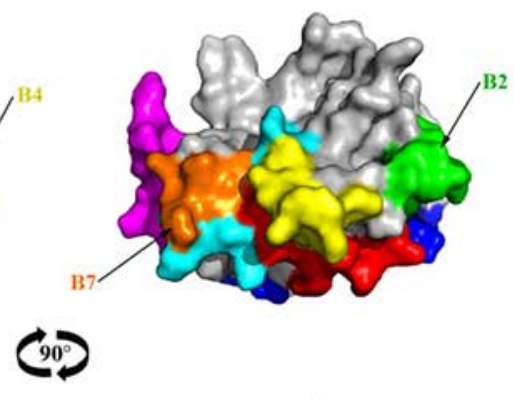

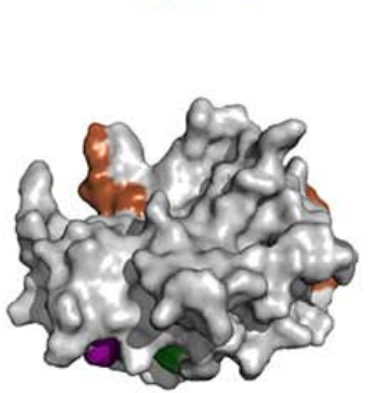

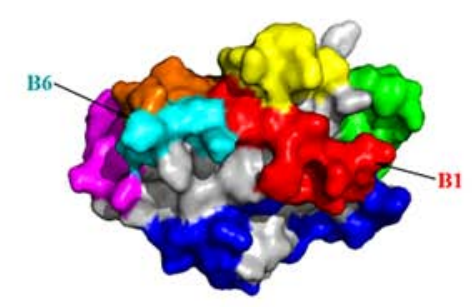

今

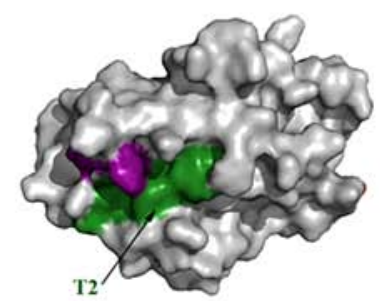

Figure 6. B cell and T cell epitope superimposition on the surface of the Per a 10 allergen structure. B1-B7 are the predicted B cell epitopes; T1-T3 are the predicted $\mathrm{T}$ cell epitopes.

we cloned and purified the American CR allergen, Per a 10, in an E. coli expression system. Per a 10 was a serine proteinase, isolated from American CR extract using a benzamidine sepharose column and characterized by immunobiochemical methods and represents a major American CR allergen, with $\mathrm{IgE}$ reactivity to $80 \%$ of sera from patients with CR allergies, as shown by skin tests and immunoblot analysis (14).

Our results showing that 9 out of $16(56.3 \%)$ of sera from patients with $\mathrm{CR}$ allergies react to Per a 10, confirmed that Per a 10 is a major allergen of the American CR. It has $<80 \%$ IgE reactivity in the Thai population (14). In this study, we confirmed the IgE reactivity of Per a 10 by a basophil activation test, which is a more advanced technique for the determination of allerginicity of allergens. It was found that Per a 10 is an active allergen of the American CR and that it is able to activate basophils which are sensitized by sera from patients with American CR allergies. The availability of recombinant allergens has increased our understanding of IgE-mediated allergies and may help to improve the diagnosis and treatment of these diseases (44). In our case, recombinant Per a 10 may be used for the further functional and clinical analysis.

Allergen-specific IgE is the key molecule for the development of allergic symptoms. The identification of B cell epitopes (IgE-binding epitopes) of an allergen is valuable for the 
accurate and safer peptide-based allergen diagnosis and immunotherapeutic agents. The in silico prediction of B cell epitopes has already become a useful tool for selecting B cell epitopes from immunological relevant proteins (45) and correlates well with the experimental approach (46). Many algorithms have been developed to predict $\mathrm{B}$ cell epitopes on a protein sequence based on the propensity values of amino acid properties of hydrophilicity, antigenicity, segmental mobility, flexibility and accessibility (47). In the present study, we predicted the B cell linear epitopes of Per a 10 allergens by the 3 sequence-based tools (DNAStar protean system, BPAP and BepiPred 1.0 server) and predicted 8 peptides (2-12, 55-67, 98-120, 125-133, 149-160, 170-182, 201-208 and 223-227) as potential B cell linear epitopes.

$\mathrm{T}$ cell epitopes are principally predicted indirectly by identifying the binding of peptide fragments to the MHC complexes. However, the binding grooves of MCH-II molecules are open at both ends, allowing various lengths of peptides to bind. On the other hand, the same MHC molecule can accommodate a variety of binding sequences. These two properties make the development of accurate predictive algorithms for MHC-class II binding complicated (40). Some algorithms have substantially improved their accuracy to predict $\mathrm{T}$ cell epitopes of immunological relevant proteins. However, most algorithms target HLA-DR molecules, but not HLA-DP and HLA-DQ molecules, even though they are important for antigen presentation. In this study, NetMHCIIpan-3.0 was applied for HLA-DR-based T cell epitope prediction of Per a 10 and finally predicted 2 peptides (83-91 and 139-147).

NetMHCII-2.2 was used for HLA-DQ-based T cell epitope prediction of Per a 10 and predicted $3 \mathrm{~T}$ cell epitope sequences, including 84-92, 139-147 and 162-170. As a result, the ultimate consensus epitope results were obtained by combining the results of the HLA-DR-based T cell epitope and HLA-DQbased $\mathrm{T}$ cell epitope. As a result, Per a 10 was predicted to have 3 T cell epitope sequences, namely 83-92, 139-147 and 162-170.

\section{Acknowledgements}

This study was sponsored by grants from the Special Fund for Forestry-Scientific Research in the Public Interest (no. 201304103), grants from the National Natural Science Foundation of China (nos. 81571568, 81400037, 31340073 and 81273274); Jiangsu Province's Key Provincial Talents Program (no. RC201170); the Priority Academic Program Development of Jiangsu Higher Education Institutions (PAPD); the Six Talents Peak projects of Jiangsu Province (to J.-F.W.).

\section{References}

1. Bernton HS and Brown H: Insect allergy preliminary studies of the cockroach. J Allergy 35: 506-513, 1964.

2. Arruda LK, Vailes LD, Ferriani VPL, Santos AB, Pomés A and Chapman MD: Cockroach allergens and asthma. J Allergy Clin Immunol 107: 419-428, 2001.

3. Sun BQ, Lai XX, Gjesing B, Spangfort MD and Zhong NS: Prevalence of sensitivity to cockroach allergens and IgE cross-reactivity between cockroach and house dust mite allergens in Chinese patients with allergic rhinitis and asthma. Chin Med J (Engl) 123: 3540-3544, 2010.

4. Thangam Sudha V, Arora N, Sridhara S, Gaur SN and Singh BP: Biopotency and identification of allergenic proteins in Periplaneta americana extract for clinical applications. Biologicals 35: 131-137, 2007.
5. He S, Zhang Z, Zhang H, Wei J, Yang L, Yang H, Sun W, Zeng $\mathrm{X}$ and Yang $\mathrm{P}$ : Analysis of properties and proinflammatory functions of cockroach allergens Per a 1.01s. Scand J Immunol 74: 288-295, 2011.

6. Wu HQ, Liu ZG, Ran PX, Zhou ZW and Gao B: Expression, purification, and immunological characterization of $\mathrm{Cr}$ PI. Protein Pept Lett 14: 881-885, 2007.

7. Mindykowski B, Jaenicke E, Tenzer S, Cirak S, Schweikardt T, Schild $\mathrm{H}$ and Decker $\mathrm{H}$ : Cockroach allergens Per a 3 are oligomers. Dev Comp Immunol 34: 722-733, 2010.

8. Tan YW, Chan SL, Ong TC, Yit Y, Tiong YS, Chew FT, Sivaraman J and Mok YK: Structures of two major allergens, Bla g 4 and Per a 4, from cockroaches and their IgE binding epitopes. J Biol Chem 284: 3148-3157, 2009.

9. Wei JF, Yang H, Li D, Gao P and He S: Preparation and identification of Per a 5 as a novel American cockroach allergen. Mediators Inflamm 2014: 591468, 2014.

10. Chen H, Yang HW, Wei JF, and Tao AL: In silico prediction of the T-cell and IgE-binding epitopes of Per a 6 and Bla g 6 allergens in cockroaches. Mol Med Rep 10: 2130-2136, 2014.

11. Yang H, Kong X, Wei J, Liu C, Song W, Zhang W, Wei W and He S: Cockroach allergen Per a 7 down-regulates expression of Toll-like receptor 9 and IL-12 release from P815 cells through PI3K and MAPK signaling pathways. Cell Physiol Biochem 29: 561-570, 2012.

12. Sookrung N, Chaicumpa W, Tungtrongchitr A, Vichyanond $P$, Bunnag C, Ramasoota P, Tongtawe P, Sakolvaree Y and Tapchaisri P: Periplaneta americana arginine kinase as a major cockroach allergen among Thai patients with major cockroach allergies. Environ Health Perspect 114: 875-880, 2006

13. Yang H, Chen H, Jin M, Xie H, He S and Wei JF: Molecular cloning, expression, IgE binding activities and in silico epitope prediction of Per a 9 allergens of the American cockroach. Int $\mathbf{J}$ Mol Med 38: 1795-1805, 2016.

14. Sudha VT, Arora N, Gaur SN, Pasha S and Singh BP: Identification of a serine protease as a major allergen (Per a 10) of Periplaneta americana. Allergy 63: 768-776, 2008.

15. Fang Y, Long C, Bai X, Liu W, Rong M, Lai R and An S: Two new types of allergens from the cockroach, Periplaneta americana. Allergy 70: 1674-1678, 2015.

16. Goel C, Kalra N, Dwarakanath BS, Gaur SN and Arora N: Per a 10 protease activity modulates CD40 expression on dendritic cell surface by nuclear factor-kappaB pathway. Clin Exp Immunol 180: 341-351, 2015.

17. Goel C, Govindaraj D, Singh BP, Farooque A, Kalra N and Arora N: Serine protease Per a 10 from Periplaneta americana bias dendritic cells towards type 2 by upregulating CD86 and low IL-12 secretions. Clin Exp Allergy 42: 412-422, 2012.

18. He W, Jimenez F, Martinez H, Harper NL, Manoharan MS, Carrillo A, Ingale P, Liu YG, Ahuja SS, Clark RA, et al: Cockroach sensitization mitigates allergic rhinoconjunctivitis symptom severity in patients allergic to house dust mites and pollen. J Allergy Clin Immunol 136: 658-666, 2015.

19. Bassirpour $\mathrm{G}$ and Zoratti E: Cockroach allergy and allergenspecific immunotherapy in asthma: Potential and pitfalls. Curr Opin Allergy Clin Immunol 14: 535-541, 2014.

20. Nielsen M, Lund O, Buus S and Lundegaard C: MHC class II epitope predictive algorithms. Immunology 130: 319-328, 2010.

21. Sookrung N, Khetsuphan T, Chaisri U, Indrawattana N, Reamtong O, Chaicumpa W and Tungtrongchitr A: Specific B-cell epitope of Per a 1: A major allergen of american cockroach (Periplaneta americana) and anatomical localization. Allergy Asthma Immunol Res 6: 325-332, 2014.

22. Lee MF, Chang CW, Song PP, Hwang GY, Lin SJ and Chen YH: IgE-binding epitope mapping and tissue localization of the major American cockroach allergen Per a 2. Allergy Asthma Immunol Res 7: 376-383, 2015.

23. Wu CH, Lee MF and Tseng CY: IgE-binding epitopes of the American cockroach Per a 3 allergen. Allergy 58: 986-992, 2003.

24. An S, Chen L, Wei JF, Yang X, Ma D, Xu X, Xu X, He S, Lu J and Lai R: Purification and characterization of two new allergens from the venom of Vespa magnifica. PLoS One 7: e31920, 2012.

25. An S, Ma D, Wei JF, Yang X, Yang HW, Yang H, Xu X, He S and Lai R: A novel allergen Tab y 1 with inhibitory activity of platelet aggregation from salivary glands of horseflies. Allergy 66: 1420-1427, 2011.

26. Sanz ML, Gamboa PM, Antépara I, Uasuf C, Vila L, Garcia-Avilés C, Chazot M and De Weck AL: Flow cytometric basophil activation test by detection of CD63 expression in patients with immediate-type reactions to betalactam antibiotics. Clin Exp Allergy 32: 277-286, 2002. 
27. Sainte-Laudy J, Vallon C and Guérin JC: Analysis of membrane expression of the CD63 human basophil activation marker. Applications to allergologic diagnosis. Allerg Immunol (Paris) 26: 211-214, 1994 (In French).

28. Yang L, Luo $\mathrm{Y}$ and Wei J: Integrative genomic analyses on Ikaros and its expression related to solid cancer prognosis. Oncol Rep 24: 571-577, 2010.

29. Yang L, Luo Y, Wei J and He S: Integrative genomic analyses on IL28RA, the common receptor of interferon- $\lambda 1,-\lambda 2$ and $-\lambda 3$. Int J Mol Med 25: 807-812, 2010.

30. Yang L, Wei $\mathbf{J}$ and He S: Integrative genomic analyses on interferon- $\lambda \mathrm{s}$ and their roles in cancer prediction. Int $\mathrm{J}$ Mol Med 25: 299-304, 2010

31. Wang M, Wei X, Shi L, Chen B, Zhao G and Yang H: Integrative genomic analyses of the histamine $\mathrm{H} 1$ receptor and its role in cancer prediction. Int J Mol Med 33: 1019-1026, 2014.

32. Ding Z, Yang HW, Xia TS, Wang B and Ding Q: Integrative genomic analyses of the RNA-binding protein, RNPC1, and its potential role in cancer prediction. Int J Mol Med 36: 473-484, 2015.

33. Li X, Yang HW, Chen $\mathrm{H}$, Wu J, Liu Y and Wei JF: In silico prediction of $\mathrm{T}$ and $\mathrm{B}$ cell epitopes of Der $\mathrm{f} 25$ in Dermatophagoides farinae. Int J Genomics 2014: 483905, 2014.

34. McGuffin LJ, Bryson K and Jones DT: The PSIPRED protein structure prediction server. Bioinformatics 16: 404-405, 2000.

35. Petersen B, Petersen TN, Andersen P, Nielsen M and Lundegaard C: A generic method for assignment of reliability scores applied to solvent accessibility predictions. BMC Struct Biol 9: 51, 2009.

36. Laskowski RA, MacArthur MW and Thornton JM: Validation of protein models derived from experiment. Curr Opin Struct Biol 8: 631-639, 1998.

37. Maganti L, Manoharan $\mathrm{P}$ and Ghoshal N: Probing the structure of Leishmania donovani chagasi DHFR-TS: Comparative protein modeling and protein-ligand interaction studies. J Mol Model 16: $1539-1547,2010$
38. Burland TG: DNASTAR's Lasergene sequence analysis software. Methods Mol Biol 132: 71-91, 2000.

39. Larsen JE, Lund $\mathrm{O}$ and Nielsen $\mathrm{M}$ : Improved method for predicting linear B-cell epitopes. Immunome Res 2: 2, 2006.

40. Yang X and Yu X: An introduction to epitope prediction methods and software. Rev Med Virol 19: 77-96, 2009.

41. Zheng LN, Lin H, Pawar R, Li ZX and Li MH: Mapping IgE binding epitopes of major shrimp (Penaeus monodon) allergen with immunoinformatics tools. Food Chem Toxicol 49: 2954-2960, 2011.

42. Karosiene E, Rasmussen M, Blicher T, Lund O, Buus S and Nielsen M: NetMHCIIpan-3.0, a common pan-specific MHC class II prediction method including all three human MHC class II isotypes, HLA-DR, HLA-DP and HLA-DQ. Immunogenetics 65: 711-724, 2013.

43. Nielsen $\mathrm{M}$ and Lund $\mathrm{O}$ : $\mathrm{NN}$-align. An artificial neural networkbased alignment algorithm for MHC class II peptide binding prediction. BMC Bioinformatics 10: 296, 2009.

44. Schmidt M and Hoffman DR: Expression systems for production of recombinant allergens. Int Arch Allergy Immunol 128: 264-270, 2002.

45. Li GF, Wang Y, Zhang ZS, Wang XJ, Ji MJ, Zhu X, Liu F, Cai XP, Wu HW and Wu GL: Identification of immunodominant Th1-type T cell epitopes from Schistosoma japonicum $28 \mathrm{kDa}$ glutathione-S-transferase, a vaccine candidate. Acta Biochim Biophys Sin (Shanghai) 37: 751-758, 2005.

46. Nair S, Kukreja N, Singh BP and Arora N: Identification of B cell epitopes of alcohol dehydrogenase allergen of Curvularia lunata. PLoS One 6: e20020, 2011.

47. Pomés A: Relevant B cell epitopes in allergic disease. Int Arch Allergy Immunol 152: 1-11, 2010. 